\author{
Haben Sie Fragen zur Abrechnung oder zur wirtschaftlichen \\ Praxisführung? Als Leser der MMW können Sie sich an unseren \\ Experten wenden: Helmut Walbert, Facharzt für Allgemein- \\ medizin und Betriebswirt aus Würzburg.
}

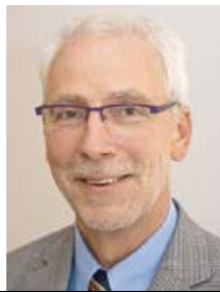

Helmut Walbert

Allgemeinarzt,

Medizinjournalist und

Betriebswirt Medizin

\title{
Die 4-3-2-1-Regel für die Chronikerziffer
}

\begin{abstract}
? Dr. A. P., Allgemeinärztin, Bayern: Ich habe meine Mitarbeiter angewiesen, bei Chronikern grundsätzlich die Nr. 03220 neben der Versichertenpauschale einzutragen. Ist dies korrekt?
\end{abstract}

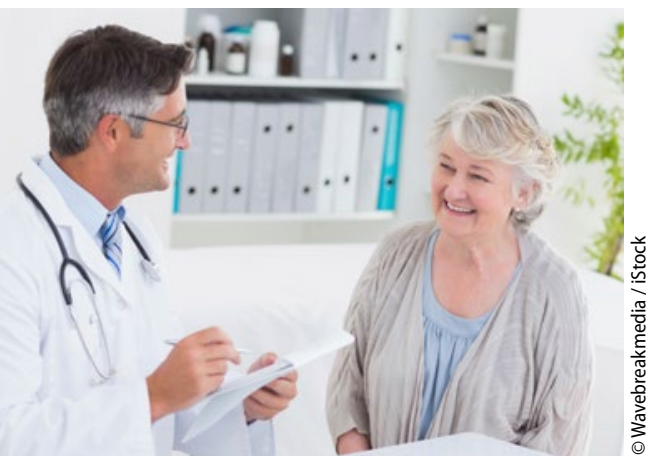

Persönliche Kontakte sind Pflicht!
MMW-Experte Walbert: Das ist in Ordnung, wenn die entsprechenden Voraussetzungen vorliegen. Für die Versichertenpauschale ist das ein persönlicher Arzt-Patienten-Kontakt, also eine direkte Interaktion am gleichen Ort. Für die Chonikerziffer gibt es die praktische „4-3-2-1-Regel“. Der Patient muss über 4 Quartale, also mindestens ein Jahr lang, an einer chronischen Erkrankung leiden. Er muss in diesen vier Quartalen mindestens 3 Kontakte zur Praxis gehabt haben. Darunter müssen 2 dokumentierte persönliche Arzt-Patienten-Kontakte sein. Und es muss sich um 1 gesicherte chronische Erkrankung handeln, also um dieselbe ICD-10-Codierung über vier Quartale.
Dies wird in vielen KVen mittlerweile durch elektronische Programme abgeprüft. Um hier nicht aufzufallen, müssen Praxen sich gut organisieren. Mit einem ersten persönlichen Arzt-Patienten-Kontakt werden die Voraussetzungen für die Chronikerziffern geschaffen. Der kleine Zuschlag nach Nr. 03221 kann schon angesetzt werden, wenn der zweite Kontakt ein telefonischer ist.

Die Kombination von Versichertenpauschale und Chronikerzuschlag spiegelt die gewollte hausärztliche Betreuung wider. Sie zu ermöglichen sollte in einer zeitgesteuerten Bestellpraxis oberste Priorität haben. Die exakte Dokumentation ist dabei wichtig, um Betrugsvorwürfen vorzubeugen.

\section{Können Hausärzte Lokalanästhesie abrechnen?}

$?$ Dr. H.-P. L., Allgemeinarzt: Ich habe mit der therapeutischen Lokalanästhesie (TLA) gute Erfahrung gemacht. Wie kann ich diese abrechnen?

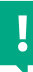
MMW-Experte Walbert: Im EBM in der Hausarztpraxis nicht. Alle Leistungen aus dem Kapitel I, die von Hausärzten abgerechnet werden können, sind in der Präambel des Hausarztkapitels unter Punkt 3 aufgezählt. Die Nr. 02360, Behandlung mit Lokalanästhetika, fehlt. Damit ist sie im Routinealltag nicht abrechenbar.
Eine Ausnahme bildet der vertragsärztliche Bereitschaftsdienst. Hier gilt die Präambel des Hausarztkapitels nicht, sodass die Nr. 02360 über den Notfallschein abrechenbar ist. Allerdings fordert die Legende mindestens drei persönliche Arzt-Patienten-Kontakte im Behandlungsfall - es müssen hier also drei persönliche Kontakte in einem Behandlungsfall im Notfalldienst sein.

Es kann sich dabei auch um eigene Patienten handeln. Wichtig ist, in diesen Fällen einen zweiten Behandlungsfall über einen Notfallschein anzulegen. Die
TLA muss nur einmal erbracht werden, öfter kann sie im Behandlungsfall aber sowieso nicht abgerechnet werden.

In der GOÄ ist die Abrechnung unkompliziert. Die Nr. 267 steht für die medikamentöse Infiltrationsbehandlung zur Verfügung. Sie wird je Sitzung abgerechnet und ist mit 80 Punkten bewertet, bringt zum einfachen Satz also 4,66 Euro ein. Werden zwei Körperregionen behandelt, kann sie auch zweimal in einer Sitzung angesetzt werden. Dies muss mit entsprechenden Diagnosen begründet werden. 